\section{What happens to an acellular dermal matrix after implanta- tion in the human body? A histological and electron microscopic study}

\author{
Martin Boháč, 1,2 Luboš Danišovič, ${ }^{2,3}$ \\ Ján Koller, ${ }^{4}$ Jana Dragúňová, ${ }^{4}$ \\ Ivan Varga ${ }^{5}$ \\ ${ }^{1}$ Department of Plastic Surgery, Faculty \\ of Medicine, Comenius University and \\ University Hospital Bratislava \\ ${ }^{2}$ Regenmed Ltd., Bratislava \\ ${ }^{3}$ Institute of Medical Biology, Genetics, \\ and Clinical Genetics, Faculty of \\ Medicine, Comenius University in \\ Bratislava \\ ${ }^{4}$ Department of Burns and \\ Reconstructive Surgery, Faculty of \\ Medicine, Comenius University and \\ University Hospital Bratislava \\ ${ }^{5}$ Institute of Histology and Embryology, \\ Faculty of Medicine, Comenius \\ University in Bratislava, Slovakia
}

\begin{abstract}
Acellular matrices are used for various purposes and they have been studied extensively for their potential roles in regenerating tissues or organs. The acellular matrix generates physiological cues that mimic the native tissue microenvironment. Acellular dermal matrix (ADM) is a soft connective tissue graft generated by a decellularization process that preserves the intact extracellular skin matrix. Upon implantation, this structure serves as a scaffold for donor-side cells to facilitate subsequent incorporation and revascularization. In breast reconstruction, ADM is used mainly for lower pole coverage and the shaping of a new breast. It helps control the positioning of the implant in the inframammary fold, and prevents the formation of contractile pseudocapsule around the breast implant. In this study, we provide a comprehensive histological description of ADM used for human breast reconstruction over the course of several months following implementation. Using immunohistochemical methods (a panel of 12 antibodies) coupled with optical and transmission electron microscopy, we confirmed that the original acellular dermal matrix became recolonized by fibroblasts and myofibroblasts, and also by various other free cells of the connective tissue (lymphocytes, macrophages and multinucleated giant cells, granulocytes, mast cells) after implantation into the patient's body.
\end{abstract}

Within the implanted ADM, there was a relatively rapid ingrowth of blood vessels. Lymphatic vessels were only detected in one case 9 months after the implantation of the ADM. These results suggest that lymphangiogenesis is a longer process than angiogenesis.

\section{Introduction}

The extracellular matrix of connective tissue is a complex biomaterial produced by different types of fixed and free connective tissue cells. The extracellular matrix acts as a support surface for cells. It is composed of many types of proteins and protein fibers, such as collagen and elastic or reticular fibers, which complex with water-soluble and insoluble molecules. The removal of cellular components from the connective tissue leaves the water-insoluble matrix, generally known as the decellularized or acellular matrix / scaffold / graft. Currently, acellular matrices are used for various purposes and they have been studied extensively for their potential roles in regenerating tissues or organs in vascular surgery, ${ }^{1}$ abdominal surgery, ${ }^{2}$ dentistry and facial surgery, ${ }^{3}$ or orthopedics. ${ }^{4,5}$ The acellular matrix generates physiological cues that mimic the native tissue microenvironment and is, therefore, more appropriate for re-functionalization and regeneration of a tissue compared with commonly used synthetic biomaterials. Thus, acellular matrices are in high demand in both basic research and clinical practice. ${ }^{6}$

An acellular tissue matrix can be prepared from any organ or tissue based upon the purpose of the research or intended clinical application, including tissues from the digestive system, urinary system, and the dermis of skin. ${ }^{7}$ Acellular dermal matrix (ADM) is a soft connective tissue graft generated via a decellularization process that preserves the intact extracellular skin matrix. Upon implantation, this structure serves as a scaffold for donor-side cells to facilitate subsequent incorporation and revascularization. ADM has been used in soft tissue reconstruction since $1995 .{ }^{8}$ Its utility has been proven in clinical practice for burn management and abdominal wall reconstruction. Since 2003, these allografts have also been used in prosthesis-based breast reconstruction. ${ }^{9}$ In breast reconstruction, ADM is used mainly for lower pole coverage and the shaping of a new breast. It helps control the positioning of the implant in the inframammary fold, as well as mimics the normal ptotic character of soft tissues in the lower pole of the neo-breast. This type of implant is more resistant to extrusion. In addition, it is less visible and
Correspondence: Assoc. Prof. Dr. Ivan Varga, Institute of Histology and Embryology, Faculty of Medicine, Comenius University in Bratislava, Spitalska 24, 81372 Bratislava, Slovakia.

Tel. +421.2.59357547.

E-mail: ivan.varga@fmed.uniba.sk

Key words: Acellular dermal matrix; breast reconstruction; histology.

Contribution: MB, study conception and experiment design, surgeries, procurement of samples, and paper drafting and revision; LD, IV study conception and experiment design, microscopic analysis and data analysis, and paper drafting and revision; JK, preparation of cellular dermal matrix and procurement of samples; JD, preparation of acellular dermal matrix. All authors read and approved the final version of the present research manuscript.

Acknowledgments: The study was supported by VEGA Research Grants No. 1/0271/17 and No. 1/0297/14 from the Slovak Ministry of Education. The authors thank Mrs. Gabriela Fujerikova, and Mrs. Viera Haraslinova for assistance during the histological preparation of specimens.

Conflict of interest: The authors declare no conflict of interest

Received for publication: 14 November 2017 Accepted for publication: 3 Janauary 2018.

This work is licensed under a Creative Commons Attribution-NonCommercial 4.0 International License (CC BY-NC 4.0).

CC Copyright M. Boháč et al., 2018

Licensee PAGEPress, Italy

European Journal of Histochemistry 2018; 62:2873 doi:10.4081/ejh.2018.2873

palpable. ADM can also minimize periprosthetic fibrosis and appears to lessen the inflammatory response associated with prosthetic devices. ${ }^{10}$ The histological process of skin acellularization at the ultrastructural level is well-known thanks to Hammer. ${ }^{11}$ Furthermore, the mechanical (tensile) properties of acellular dermal grafts have been studied intensively. ${ }^{7}$ Much less is known about the processes of integration and subsequent morphological changes in the ADM implanted into host tissues, such as re-cellularization and re-vascularization. Until now, the histology of ADM host tissue integration has been studied in various animal models, including mice, ${ }^{12}$ rats, ${ }^{13,14}$ rabbits, ${ }^{15}$ and pigs. ${ }^{16,17}$ Similar histological studies of human ADM implants are limited, and only one article on this topic has been published by Bullocks. ${ }^{18}$

This study provides a comprehensive histological description of ADM used for 
human breast reconstruction over the course of several months following implementation. Using immunohistochemical methods coupled with optical and transmission electron microscopy, we describe host cell populations that invade fully integrated ADM after implantation.

\section{Materials and Methods}

The Central Tissue Bank (CTB) of the Department of Burns and Reconstructive Surgery at the University Hospital in Bratislava, Slovakia provided us with ADM. Freshly procured allogeneic cadaver skin grafts or cryopreserved allogeneic skin grafts from the CTB served as tissue sources for ADM preparation. A new method of allogeneic cadaveric human dermis decellularization (based on the action of a proteolytic enzyme in combination with distilled water) was developed at the CTB and was previously published by Dragúňová et al. ${ }^{19}$ (Figure 1). In our study, we analyzed samples from 22 patients (26 breasts), who underwent delayed-staged breast reconstructions using ADM to protect the lower poles of temporary tissue expanders, followed by permanent prosthesis implantations. During the expander exchange procedure, histological samples were obtained without inducing additional morbidity. Biopsies were harvested during expander to implant change. They were

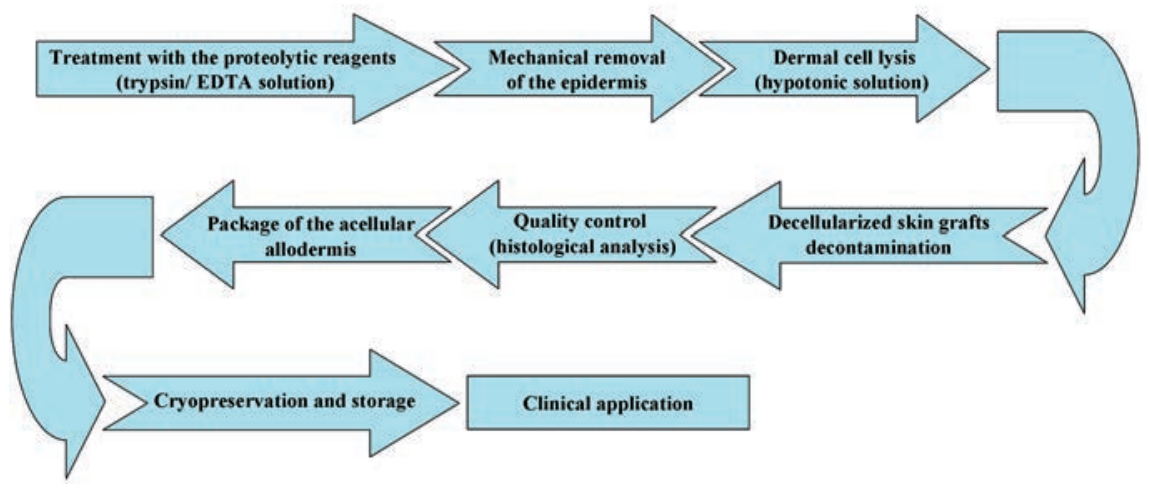

Figure 1. Schematic overview of acellular dermal matrix preparation.

Table 1. Basic characteristics of the study group of patients and description of samples of ADM used during post mastectomy breast reconstructions.

\begin{tabular}{ll}
\hline Total patients & 22 \\
Total breasts & 26 \\
\hline Patients with unilateral breast reconstruction & 18 \\
Patients with bilateral breast reconstruction & 4 \\
\hline Average age (years) & 48 (range from 30 to 69 ) \\
Average size of ADM & $273 \mathrm{~cm}^{2}$ (range from 155 to $\left.665 \mathrm{~cm}^{2}\right)$ \\
\hline Average operation duration & 79 minutes (range from 60 to 105 minutes) \\
Average size of expansion & $389 \mathrm{cc}$ (range from 155 to $690 \mathrm{cc}$ ) \\
\hline Average time for expander replacement by permanent & 5.9 months (range from 3 to 9 months)
\end{tabular}

implant - expansion time

Complications, as seroma or hematoma formation $\quad 0$

or capsular contracture

ADM, acellular dermal matrix.

Table 2. Characteristics of all monoclonal antibodies used (in alphabetical order).

\begin{tabular}{|c|c|c|c|}
\hline Antibodies against & Short description & Manufacturer, code & Dilution \\
\hline$\alpha$-smooth muscle actin & $\begin{array}{l}\text { Belongs to the microfilament system of smooth muscle cells, } \\
\text { myofibroblasts, and myoepithelial cells }\end{array}$ & Dako, MO851 & $1: 200$ \\
\hline CD20 & $\begin{array}{l}\text { Antigen located on the surface of B lymphocytes and is lost shortly before } \\
\text { the terminal plasma cell stage }\end{array}$ & Dako, MO755 & 1:100 \\
\hline CD34 & $\begin{array}{l}\text { Transmembrane protein expressed on immature hematopoietic progenitor } \\
\text { cells, capillary endothelial cells, and embryonic fibroblasts }\end{array}$ & Dako, M7165 & 1:150 \\
\hline CD45RO & A surface marker for activated T cells & Dako, MO742 & 1:200 \\
\hline CD68 & $\begin{array}{l}\text { Strongly expressed in cytoplasmic granules, and weakly on the surface } \\
\text { of macrophages, monocytes, neutrophils, basophils and NK-cells }\end{array}$ & Dako, MO876 & 1:150 \\
\hline Cytokeratin AE1/AE3 & $\begin{array}{l}\text { A cocktail of two antibodies that bind two epitopes present on the majority } \\
\text { of epithelial cytokeratins; labels the cytoskeleton of epithelial cells }\end{array}$ & Dako, M3515 & 1:300 \\
\hline Desmin & $\begin{array}{l}\text { Forms a cytoskeletal network across the muscle fibers; specific for smooth } \\
\text { muscle cells and striated muscle fibers }\end{array}$ & Dako, MO760 & 1:100 \\
\hline Podoplanin, clone D2-40 & $\begin{array}{l}\text { Expressed in the endothelium of lymphatic vessels, but not in blood } \\
\text { endothelial cells }\end{array}$ & Dako, M3619 & 1:150 \\
\hline S100 protein & $\begin{array}{l}\text { Present in cells derived from the neural crest (e.g., Schwann cells), } \\
\text { but also described in dendritic cells }\end{array}$ & Dako, Z0311 & 1:1000 \\
\hline Vimentin & $\begin{array}{l}\text { Cytoskeletal protein of the type III intermediate filament found in the cells } \\
\text { of mesenchymal origin }\end{array}$ & Dako, IR630 & Diluted by manufacturer \\
\hline Von Willebrand factor (factor VIII) & Expressed in endothelial cells and megakaryocytes & Dako, A0082 & $1: 100$ \\
\hline
\end{tabular}


obtained by elliptical excision in full thickness way of surrounding tissue around expander. The average period of time between expander placement and permanent implant replacement was 5.9 months, but this ranged from 3 to 9 months (Table 1). As a negative control, acellular dermal matrix was used before implantation and histologically examined. As a positive control, small biopsies of a native skin removed during surgery were used for transmission electron microscopy.

The Ethical Committee of the University Hospital Bratislava approved the study protocol UHB/006/2015/MB, and all patients signed informed consent before participating in the study. The clinical study was also registered in the ClinicalTrials.gov database (NCT02835781).

Biopsies of explanted ADMs from the patients were processed for light microscopy. Briefly, samples were fixed in $10 \%$ formalin solution, dehydrated, cleared, and embedded in paraffin according to the established guidelines of the Institute of Histology and Embryology, Comenius University in Bratislava, Slovakia. Paraffin blocks were sectioned in 5 - $\mu$ m-thick sections, and stained with hematoxylin and eosin, Sirius red as a method for collagen fibers determination FLUKA $^{\mathrm{TM}}$, Buchs, Switzerland) and Resorcin-Fuchsin stain
(Mikrochem ${ }^{\circledR}$, Pezinok, Slovakia), for visualization of elastic fibers. In addition, immunohistochemical staining was performed on another set of sections to examine a variety of cell populations. To do this, slides were stained with eleven different primary antibodies, including von Willebrand factor (factor VIII), CD20, CD34，CD45RO, CD68, cytokeratin AE1/AE3, desmin, vimentin, S100 protein, $\alpha$-smooth muscle actin, and podoplanin (D2-40). A description of these antibodies is shown in Table 2. The primary antibodies were used according to the manufacturers' protocols. For visualization, we used the EnVisionTM FLEX Detection System
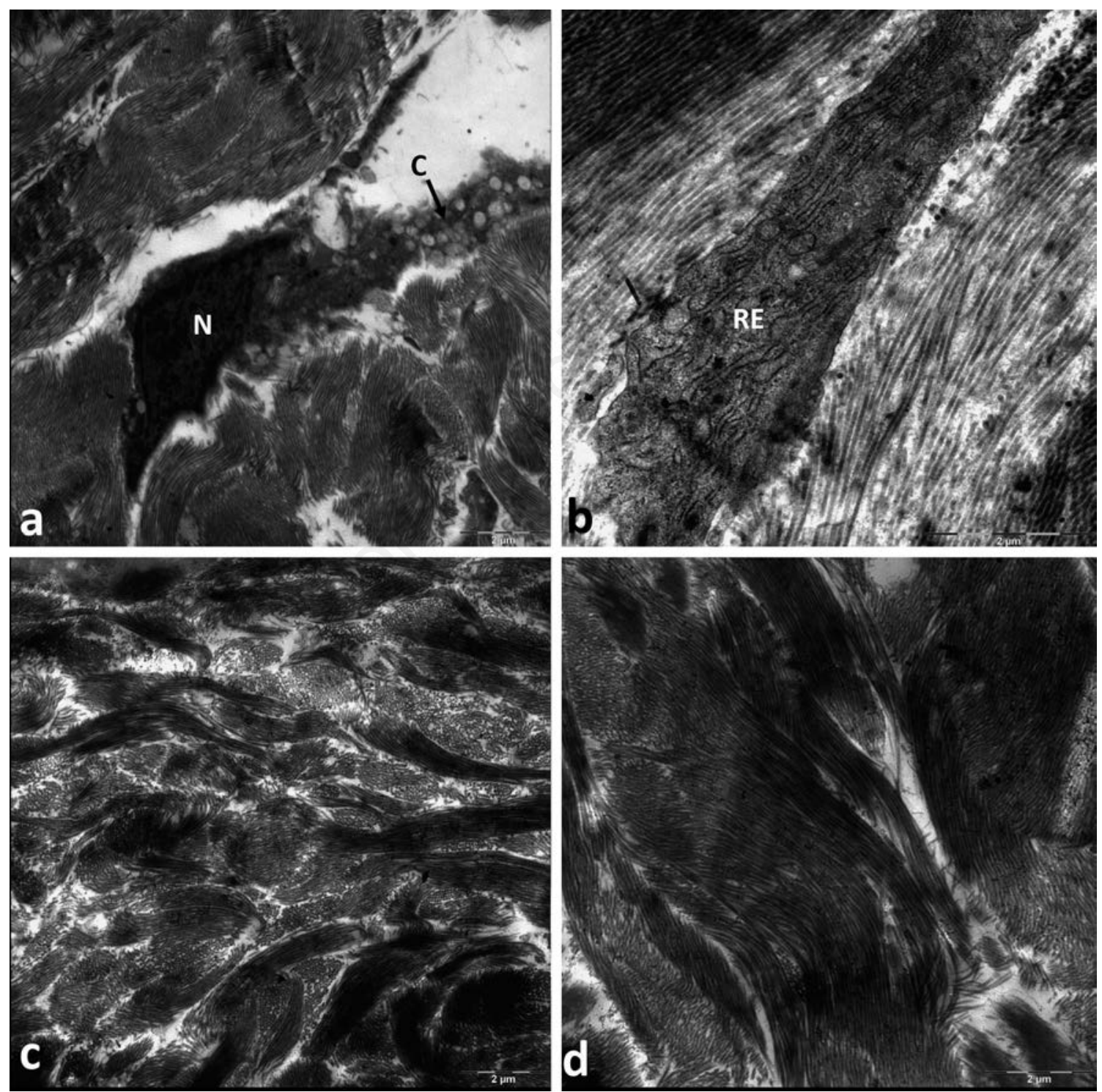

Figure 2. Native (a,b) and acellular (c,d) dermis visualized by transmission electron microscopy. a) Fibroblast with predominantly euchromatic nucleus $(\mathrm{N})$ located between bundles of collagen fibers. The cytoplasmic process $(\mathrm{C})$ is filled with secretory vesicles; magnification: 7100x. b) Representative image of the cytoplasm of fibroblasts located between collagen fibers. Numerous sacs of rough endoplasmic reticulum (RE) are visible; magnification: $14,000 x . c, d)$ Within the acellular dermal matrix, only bundles of collagen fibers are visible, but no cells; magnification: $7100 x$ and $8900 x$, respectively. 
(DAKO, Glostrup, Denmark) with diaminobenzidine as a chromogen. To orient the tissues, cell nuclei were stained with Mayer's hematoxylin in dark blue. The sections were examined on a LEICA DM2500 light microscope, and microphotographs were obtained using a LEICA DFC290HD digital camera. Additionally, granulocytes were identified via histochemical detection of Naphthol AS-D Chloroacetate-Esterase (Sigma-Aldrich ${ }^{\circledR}$, St. Louis, MO, USA).

Tissue biopsy samples $\left(1-2 \mathrm{~mm}^{3}\right)$ from the explanted ADM were obtained immediately after interruption of the blood supply during reconstructive surgeries. As controls, acellular dermal matrix before implantation and one sample of the native dermis of the skin were examined. Tissues were fixed in $3 \%$ glutaraldehyde in phosphate-buffered solution for $3 \mathrm{~h}$ at room temperature. Afterwards, samples were rinsed three times in $0.1 \mathrm{M}$ sodium phosphate-buffered solution $(\mathrm{pH} 7.3)$ and post-fixed in $1 \%$ osmium tetroxide at $4^{\circ} \mathrm{C}$. Dehydration into $100 \%$ ethanol using a graded ethanol series was performed, followed by tissue clearing in epoxypropane. Tissues were placed in a mixture of resin (Durcupan ACM, Fluka, Switzerland) and epoxypropane (1:1) at room temperature for several hours. Samples were embedded in Durcupan ACM and polymerized at $60^{\circ} \mathrm{C}$ for $72 \mathrm{~h}$. Subsequently, the specimens were cut on an ultramicrotome. To obtain the highest contrast for the ultrathin sections $(70-90 \mathrm{~nm})$, uranyl acetate with Reynolds' solution was used. Samples were visualized on the FEI Morgagni 268D TEM and imaged using a 4 MPxCCD digital camera.

\section{Results}

Transmission electron microscopy of native skin and ADM before implantation

The reticular dermis is typically a dense, irregular connective tissue. Inside the native dermis, we only found some fibroblasts between huge bundles of collagen fibers that crossed each other in different planes. These cells of the connective tissue manufacture the extracellular matrix and their cytoplasm was observed to be filled with rough endoplasmic reticulum and secretory vesicles (Figure 2 a,b). In samples from the acellular dermal matrix obtained after decellularization but before implantation inside a patients' body, we found no cells and only huge bundles of collagen fibers instead (Figure $2 \mathrm{c}, \mathrm{d}$ ). No changes in the collagen fibers were observed to be related to the process of decellularization. Furthermore, the collagen fibers inside the acellular dermal matrix displayed typical cross bands that were similar to the intact fibers within the native dermis. The decellularization process described by us effectively eliminates the cells of the connective tissue, but does not damage the structure or organization of the collagen fibers.

\section{Transmission electron microscopy of ADM after implantation}

The ultrastructure of the implanted ADM after several months are presented (Figure 3). After several months, the implanted ADM acquired a loose connective tissue appearance, and heterogeneous cell populations associated with it. The most abundant cells were fibroblasts (Figure 3 $\mathrm{a}, \mathrm{b})$. These cells were extremely proteosynthetically active. These cells possessed a large, pale nucleus (suggesting a significant amount of euchromatin), a well-developed nucleolus, and dilated cisterns and sacs of rough endoplasmic reticulum. They were much more active in the manufacturing of extracellular matrix than those from the native dermis. We also found typical signs of active angiogenesis (Figure 3a). The newly formed capillaries consisted of more cuboidal endothelial cells compared to the squamous endothelial cells in native connective tissue. We also detected cells of lymphoid and myeloid lineages, as well as plasma cells/large lymphocytes, macrophages, granulocytes, and mast cells (Figure $3 \mathrm{c}$-f). These results suggest that the ADM became completely colonized by numerous types of cells, which is a characteristic phenomenon observed in loose connective tissues.

\section{Light microscopy of ADM before implantation}

Representative images of tissue samples of ADM before implantation stained with hematoxylin and eosin, Sirius red and resorcin-fuchsin stain are shown in Figure 4. The process of decellularization destroyed all of the cells in the dermis. Figure 4 a,b shows the structure of the ADM before implantation. These samples were composed only of bundles of collagen fibers, and no cells were present. Sirius red staining (Figure 4c) demonstrates the presence of intact bundles of collagen fibers, but the elastic fibers visualized by resorcin-fuchsin stain are fragmented (Figure 4d). Following decellularization, we found no detectable intact CD34 of Factor VIII positive endothelial cells inside native ADM. We observed only dispersed CD34 and Factor VIII positive puncta in the decellularized tissue, which were probably remnants of osmotically ruptured cells that had been removed (Figure 4 e-f).

\section{Light microscopy of ADM after implantation}

Figure 5 shows the morphology of the ADM after implantation by hematoxylin and eosin, Sirius red and resorcin-fuschsin staining. Around newly formed blood vessels we found thinner, wavy appearance collagen fibers, which represented probably newly manufactured fibers (Figure 5a). Also the elastic fibers were longer and less fragmented which may be a sign of new formation of elastic fibers (Figure 5b). Although the ADMs spent several months within the host tissue, the difference between the host connective tissue and the implanted ADM was still obvious. Even though capillaries and small blood vessels were visible inside the ADM, the vascularization of this connective tissue was less noticeable than the vascularization of the loose connective tissue between the chest wall (pectoralis skeletal muscles) and the implanted ADM (Figure 5c). Regardless, the presence of fibroblasts and blood vessels within the originally acellular matrix was undeniable. We found morphological signs of angiogenesis within the ADM: endothelial cells of smaller blood vessels and capillaries were cuboidal in shape and not squamous, which is normally seen (Figure 5d). On the border between the implanted ADM and the native connective tissue, we detected multinucleated giant cells (Figure 5f), which were likely a response to the presence of a foreign body, such as the remnants of suture materials.

Immunohistochemistry and histochemistry revealed the presence of blood and lymph vessels, as well as diverse cell types inside the implanted ADM. We detected blood capillaries and even smaller vessels in the ADM after implantation by using three different antibodies (Figure $6 \mathrm{a}-\mathrm{c}$ ), and in one case, we observed lymphatic vessels (Figure 6d). We detected endothelial cells lining the blood and lymph vessels, and smooth muscle cells formed portions of the walls of vessels. The amount and density of the blood vessels were lower in the ADM compared to the patient's adjacent tissue. Surprisingly, the high density of newly formed blood vessels was observed in the marginal part of the ADM on the side opposite the breast implant. These dense blood vessels were located just below the pseudocapsule, which was composed of a thick layer of synovial metaplastic cells that formed on the surface as a result of the pressure and movement of the implant. Podoplanin (D2-40) is a specific marker for the endothelial cells of lymph vessels. We observed lymph vessels only in one case in which a patient had an ADM implanted for the longest time period (9 months). In the other samples, we failed to confirm the 

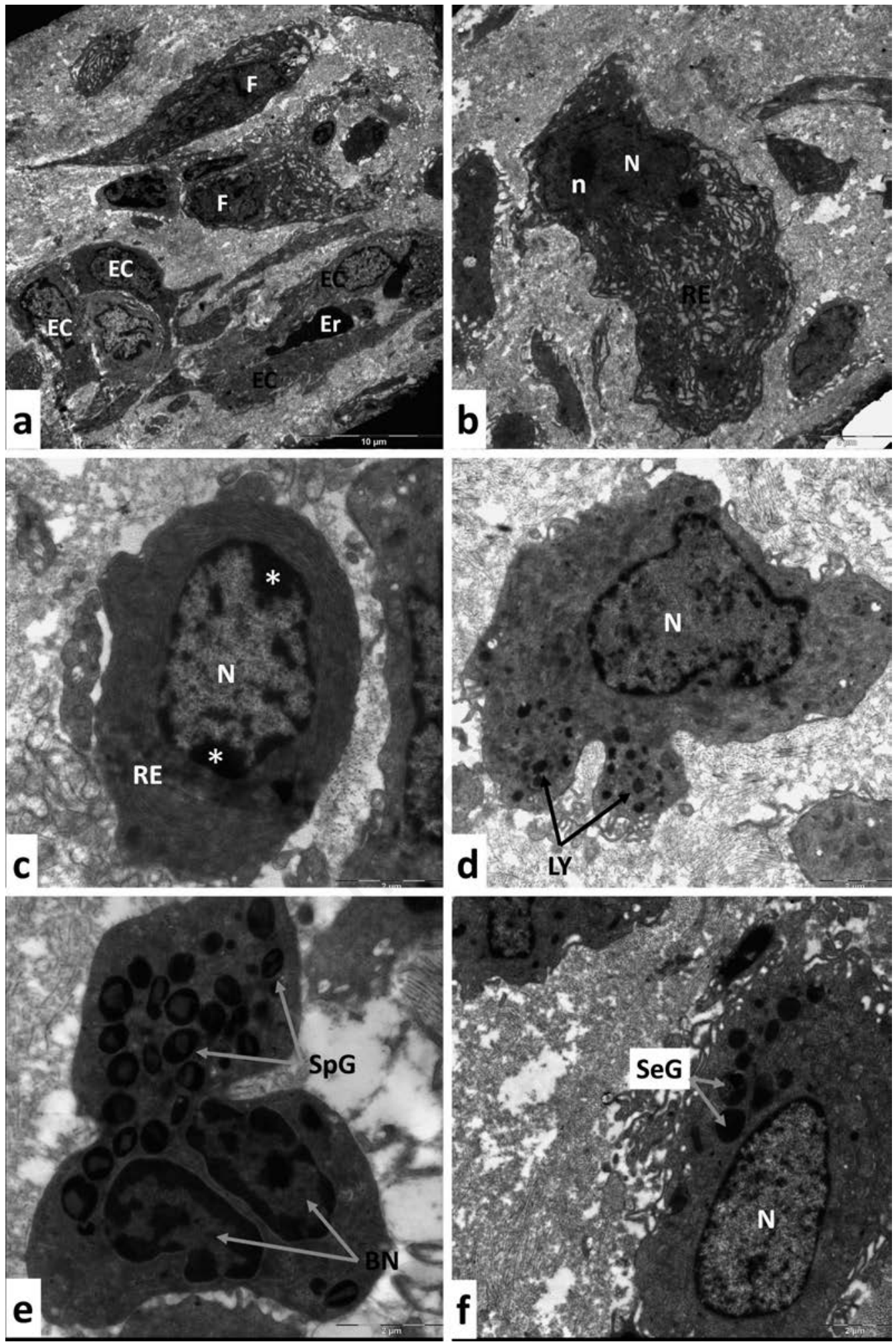

Figure 3. Heterogeneous cellular populations inside the previously acellular dermal matrix approximately half year after implantation visualized by transmission electron microscopy. a) Process of neovasculogenesis. Higher (cuboidal) endothelial cells (EC) form new capillaries; erythrocytes $(\mathrm{Er})$ are visible inside the lumen; around the capillaries, fibroblasts (F) are present; magnification: 2800x. b) Fibroblast show typical morphological signs of high proteosynthetic activity, such as a euchromatic nucleus (N), a noticeable nucleolus (n), and dilated sacs and cisterns of rough endoplasmic reticulum (RE); magnification: 3500x. c) Large lymphocyte or plasma cell with typical nucleus $(\mathrm{N})$ showing "clock-face" condensation of heterochromatin $\left(^{*}\right)$ inside the nuclear envelope; the cytoplasm contains welldeveloped rough endoplasmic reticulum (RE); magnification: 11,000x. d) Macrophage with an irregularly shaped nucleus (N) and numerous electron-dense lysosomes (LY); magnification: 7100x. e) Eosinophilic granulocytes with bilobed nucleus (BL) and numerous specific granules (SpG) show the presence of crystalloid within through the cytoplasm; magnification: 11,000x. f) Mast cell with oval nucleus $(\mathrm{N})$ and secretory granules $(\mathrm{SeG})$ after partial degranulation; magnification: $7100 \mathrm{x}$. 
presence of newly formed lymphatic vessels. This also meant that the development of new blood vessels in the acellular tissue occurred earlier and faster than the de novo formation of the lymphatic vessels.

Inside the implanted ADM (Figure 7), we also observed CD20-positive B-lymphocytes. These occasionally generated smaller lymphoid nodules on the border between the $\mathrm{ADM}$ and the native connective tissue, but we could not detect B-lymphocytes inside the ADM. We also observed
CD45RO-positive T-lymphocytes (which were evenly dispersed throughout the ADM), CD68-positive macrophages (which were dispersed through the whole ADM) and multinucleated giant cells (which were present on the border between the ADM and
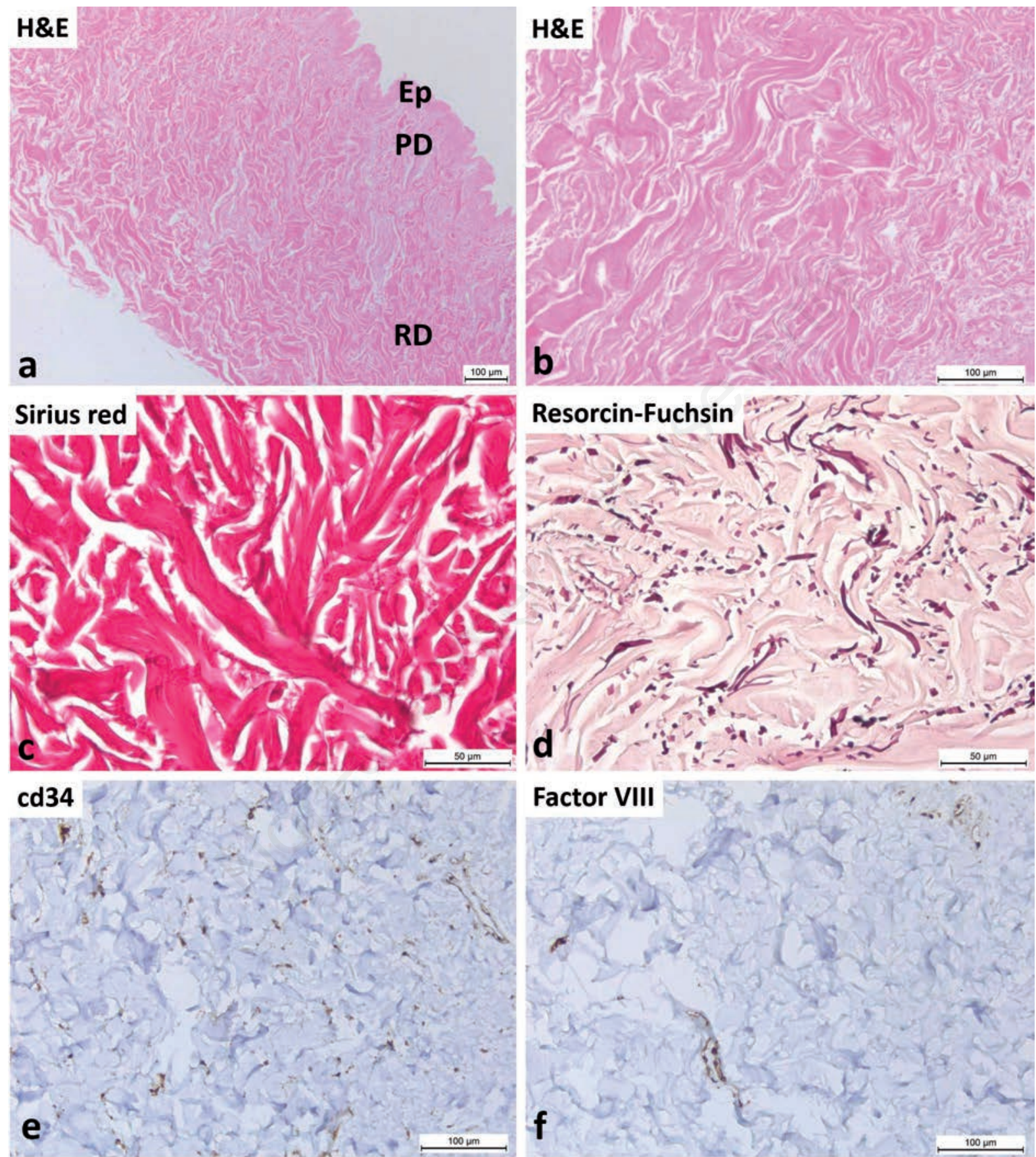

Figure 4. Acellular dermal matrix before implantation. a) View of the decellularized skin at low magnification; Ep, remnants of epidermis; PD, papillary dermis; RD, reticular dermis. b) In the decellularized dermis, only collagen fibers are visible and no cells. c) Demonstration of the collagen fibers by Sirius red stain. d) Fragmentation of elastic fibers after the decellularization procedure (resorcin-fuchsin stain). e) Immunohistochemical identification of blood vessels. Inside the decellularized dermis, there are no CD34-positive cells. Diffuse and faint reactive staining for the CD34 antigen (probably from osmotically ruptured and removed cells) is present. $\mathrm{f}$ ) Inside the decellularized dermis, there are no detectable Factor VIII-positive endothelial cells. Only diffuse and faint reactive staining for the factor VIII antigen (probably from osmotically ruptured and removed cells) is present. 
the native connective tissue, probably as a reaction to foreign bodies like surgical sutures), and chloracetate-esterase-positive granulocytes. The most numerous cells inside the ADM were vimentin-positive spindle-shaped cells, which were likely fibroblasts and fibrocytes. We also detected spindle-shaped cells that were positive for $\alpha$-smooth muscle actin, which were probably myofibroblasts and smooth muscle cells in the wall of blood vessels. All of these cells create a cellular microenvironment for normal connective tissues. In contrast, within the implanted ADM, we found no cytokeratin-positive (epithelial) cells, S100-positive myelinated nerve fibers, or vimentinpositive cells.
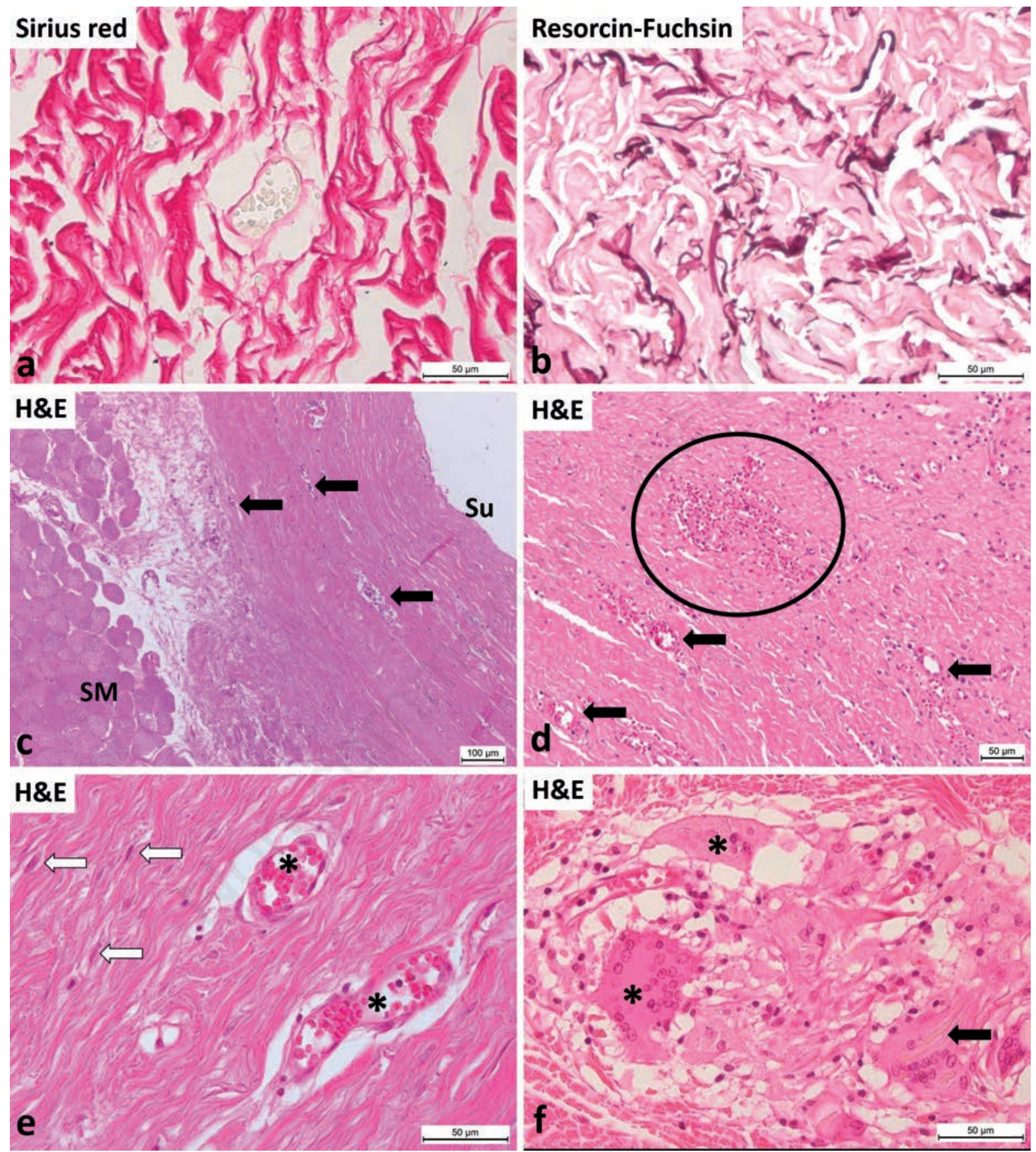

Figure 5. Acellular dermal matrix several months after implantation during breast reconstruction. a) Around blood vessels newly formed collagen fibers are visible, which are paler, thinner and they do not form so huge bundles as inside the ADM before implantation (Sirius red stain). b) Demonstration of elastic fibers, which are longer and less fragmented as inside the ADM before implantation (resorcin fuchsin stain). c) Explanted ADM after several months inside the human body, between skeletal muscle fibers (SM) and the surface (Su); inside some smaller blood vessels, capillaries are visible (arrows). d) ADM after explant procedure at high magnification; smaller vessels / larger capillaries (arrows) are visible; inside the ellipse a rich capillary bed. e) Detailed view of the re-vascularized ADM showing two smaller thin-walled vessels / capillaries (asterisks); between collagen fibers, the nuclei of fibroblasts are visible (white arrows). f) Multinucleated giant cells (asterisks) and likely macrophages are occasionally present on the border between the implanted ADM and the patients' normal connective tissues; these giant cells phagocytose suture residues (arrow). 


\section{Discussion}

In this study, we confirmed that the original acellular dermal matrix became recolonized by fibroblasts and myofibroblasts, and also by various other free cells of the connective tissue (lymphocytes, macrophages, granulocytes, mast cells) after implantation into the patient's body. Within the implanted ADM, there was a relatively rapid ingrowth of blood vessels. Lymphatic vessels were only detected in one case 9 months after the implantation of the ADM. In addition, these lymphatic vessels formed only under the pseudocapsule that developed around the breast implant. These results suggest that lymphangiogene- sis is a longer process than angiogenesis. To our knowledge, this is the second published article that has performed a histological analysis of the incorporation of the ADM into the surrounding tissue during breast reconstruction. The first article was published by Bullocks. ${ }^{18}$ He observed the presence of fibroblasts and blood vessels inside the ADM (although the number of cells and vessels were less than those of the host tissue), the invasion of giant cells in response to a foreign body (likely suturing material), and the formation of a pseudocapsule on the side of the implant facing the expander. In our study, we have confirmed all of Bullocks' results and we have also extended these results by using electron microscopy and immunohistochemistry on biopsy samples taken from patients. Using this approach, we described the revascularization of the ADM, as well as observed the presence of heterogeneous cells within the ADM.

For breast surgery, there are several clinical scenarios in which ADMs seem to be useful and may improve outcomes compared to traditional techniques. The ADM serves as a unique tool for treating a postmastectomy prosthetic breast reconstruction patient. Using the ADM to create a pocket for the tissue expander and the subsequent implant eliminates the need for elevating the serratus anterior muscle or the rectus abdominis fascia for prosthesis coverage.

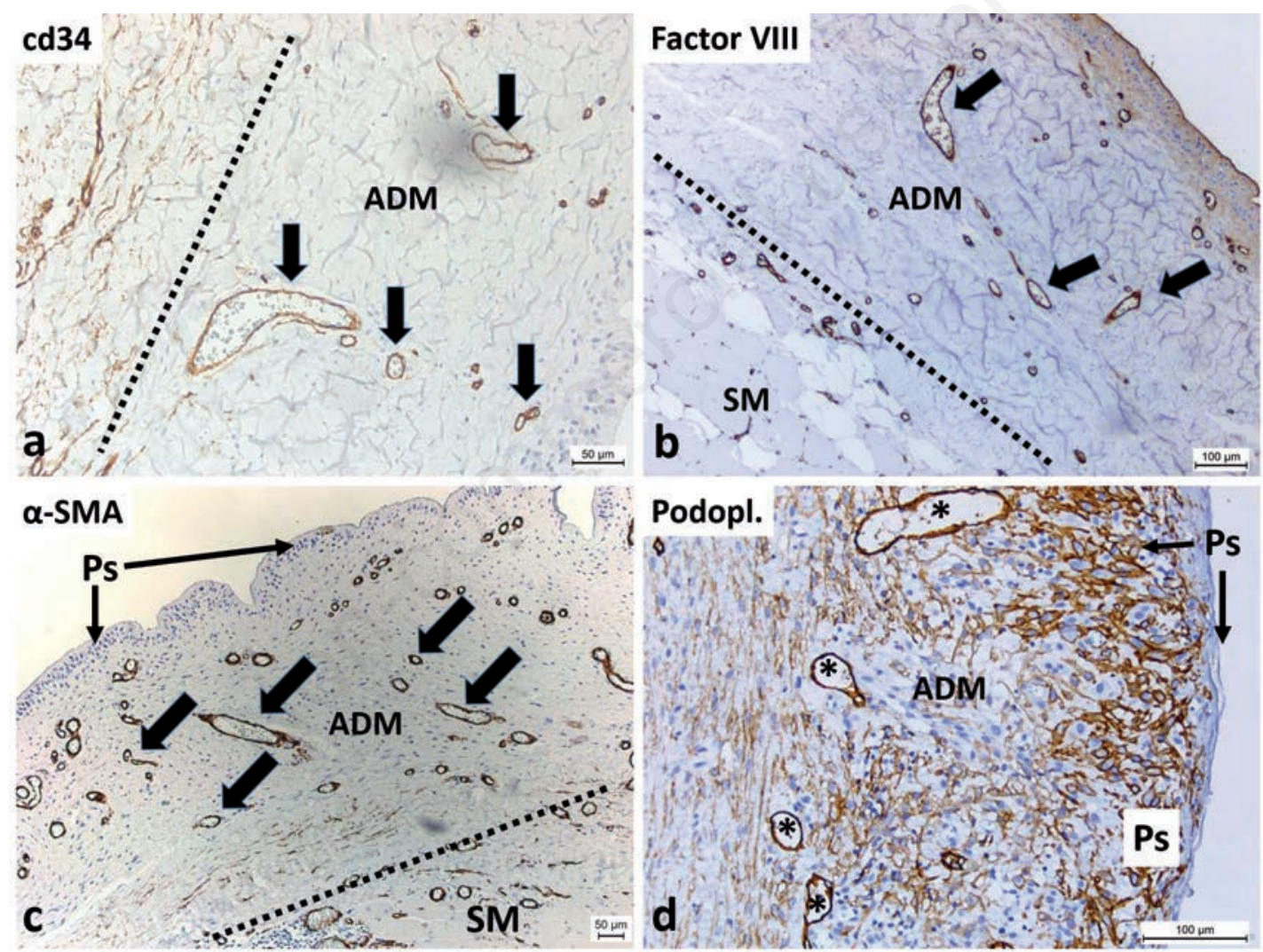

Figure 6. Immunohistochemical identification of blood and lymph vessels inside the ADM several months following implantation. a) The line indicates the border between host connective tissue (left side) and the ADM after implantation; within the ADM are visible blood vessels (with CD34-positive endothelial cells, arrows), but the occurrence of other CD34-positive cells (e.g., fibroblasts) is less frequent. b) The line indicates the border between the patients' own connective tissue (left and down) and the ADM after implantation; endothelial cells (Factor VIII-positive) lining the blood vessels are also present inside the ADM (arrows); the patient's own tissue contains skeletal muscle fibers (SM); at the surface between the ADM and the breast implant, a pseudocapsule (Ps) from cells undergoing synovial metaplasia is visible. c) The line indicates the border between host connective tissue (right and down) and the ADM after implantation; $\alpha$-smooth muscle actin-positive cells (smooth muscle fibers) form a part of the wall of the blood vessels inside the ADM (arrows); the patient's tissue contains skeletal muscle fibers (SM); at the surface between the ADM and breast implant, a capsular synovial metaplasia forming a pseudocapsule (Ps) is visible, harboring numerous blood vessels associated with this pseudocapsule. d) In one case, after the longest period of ADM implantation (more than 9 months), lymphatic vessels are observable (asterisks, endothelial cells of lymph vessels are podoplanin-positive); the surface-lining podoplanin-positive cells are synovial metaplastic cells that form a pseudocapsule (Ps) around the breast implant. 
This potentially decreases postoperative pain and local muscular deficits, as well as reducing operation times. Appropriate patient selection, proper surgical techniques, and adjusted postoperative management are critical for the success of ADMassisted reconstruction, including minimiz- ing the risk of complications. Known patient risk factors for implant-based reconstruction, such as obesity and smoking, also apply to ADM-assisted reconstruction and should be considered during patient selection. . $^{10,20,21}$

Revascularization of ADM during an experimental reconstruction of abdominal wall defects in a rabbit model was histologically verified by Menon et al. ${ }^{15}$ Although it is clear that ADM should be revascularized by host tissues, the precise mechanisms underpinning $\mathrm{ADM}$ incorporation remain unknown. Most of authors described this

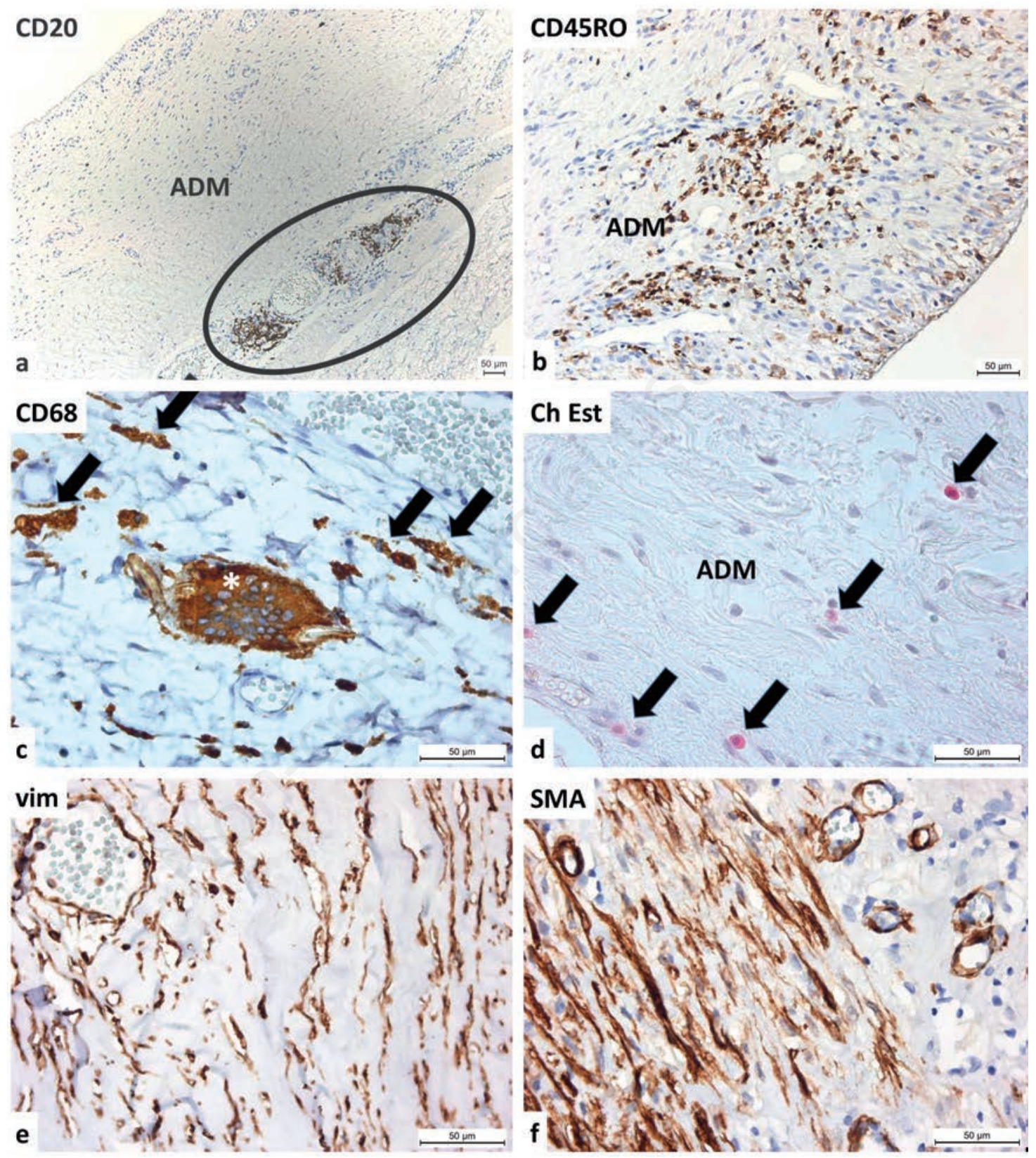

Figure 7. Heterogeneous cell populations inside the implanted ADM. a) At the border between the ADM and host's connective tissue, an accumulation of CD20-positive B-lymphocytes is observed; at the surface, synovial metaplastic cells form a pseudocapsule. b) The implanted ADM is housed by CD45RO-positive T-lymphocytes; T-lymphocytes are also present within the pseudocapsule formed by synovial metaplastic cells. c) At the border between the ADM and the host tissue, numerous macrophages (arrows) and occasion multinucleated giant cells (asterisk) are present (both are CD68-positive). d) The Naphthol AS-D Chloroacetate-Esterase reaction detects granulocytes inside the connective tissue of the ADM. e) Spindle-shaped, vimentin-positive cells (mostly fibroblasts, fibrocytes and blood endothelial cells) are observed. f) Spindle-shaped cells positive for $\alpha$-smooth muscle actin (mostly myofibroblasts, pericytes and vascular smooth muscle cells) are observed. 
process of integration as a form of normal wound healing. ${ }^{22,23}$ Wong et al. ${ }^{22}$ monitored ADM infiltration by various cells for 2 weeks in rats. The first cells that invaded the ADM were myofibroblasts and endothelial cells of vessels. Lymphatic vessels developed even at the end of the observed period. Similarly, Cherubino et al. ${ }^{14}$ describes lymphangiogenesis in ADM in rats. However, it should be emphasized that the processes of angiogenesis and lymphangiogenesis is different in rats, and it is apparently faster than in larger animals or in humans. For example, when a pig is used as a model animal, vessels are present within the ADM even at 8 weeks following implantation. ${ }^{16}$ Here, we have confirmed that blood vessel formation occurs in ADMs in humans, and in only one case were we unable to confirm the presence of lymphatic vessels.

The process of integrating ADM into the host tissue is also dependent on the type and method of ADM preparation. Capito et al. ${ }^{13}$ applied four different commercially available acellular dermal matrices from four different manufacturers to the rat body. They found that the rate of revascularization and the number of inbred cells differed significantly among individual ADMs. Similarly, Carruthers et al. ${ }^{24}$ described distinct remodeling characteristics in a porcine model of AMDs from different manufacturers. As part of our research, ADMs were prepared using a simple and inexpensive method. Although we could not compare our results to those of the authors who performed their experiments on animal models, we have found similar processes at work during ADM integration in the human body. First, we observed significant angiogenesis and the ingrowth of fibroblasts and myofibroblasts. Subsequently, we detected various free connective tissue cells of the lymphoid and myeloid lineages. The process of integrating ADMs into the surrounding tissue will be accelerated in the future by seeding mesenchymal stem cells (isolated from adipose tissue, for example) onto the ADM prior to its application. ${ }^{17}$

Based on animal research, it is known that after implantation into host tissue, ADMs are invaded by blood and lymphatic vessels, as well as connective tissue cells (such as fibroblasts and myofibroblasts). However, wound healing and ADM integration in animal models may not extend to what happens in the body of a human. To our knowledge, this is one of the first histological and electron microscopic studies examining the integration of ADMs into human tissues. We have found that ADMs are well-vascularized after several months and become colonized by numerous fibroblasts and myofibroblasts, as well as lympho- cytes, macrophages, granulocytes, and mast cells. However, the formation of new lymphatic vessels within ADMs has not been confirmed. We only observed lymphatic vessels that developed on the surface underneath the pseudocapsule, which was likely a response to implant pressure.

\section{References}

1. Kubíková T, Kochová P, Brázdil J, Špatenka J, Burkert J, Králíčková M, et al. The composition and biomechanical properties of human cryopreserved aortas, pulmonary trunks, and aortic and pulmonary cusps. Ann Anat 2017;212: 17-26.

2. Porzionato A, Sfriso MM, Macchi V, Rambaldo A, Lago G, Lancerotto L, et al. Decellularized omentum as novel biologic scaffold for reconstructive surgery and regenerative medicine. Eur $\mathrm{J}$ Histochem 2013;57:e4.

3. De Ponte FS, Cutroneo G, Falzea R, Rizzo G, Catalfamo L, Favaloro A et al. Histochemical and morphological aspects of fresh frozen bone: a preliminary study. Eur J Histochem 2016;60:2642.

4. Rampichova M, Chvojka J, Jencova V, Kubikova T, Tonar Z, Erben J, et al. The combination of nanofibrous and microfibrous materials for enhancement of cell infiltration and in vivo bone tissue formation. Biomed Mater 2017. doi: 10.1088/1748-605X/aa9717. [Epub ahead of print].

5. Gögele C, Schwarz S, Ondruschka B, Hammer N, Schulze-Tanzil G. Decellularized iliotibial band recolonized with allogenic homotopic fibroblasts or bone marrow-derived mesenchymal stromal cells. Methods Mol Biol 2017. doi: 10.1007/7651 201730 . [Epub ahead of print].

6. Rijal G. The decellularized extracellular matrix in regenerative medicine. Regen Med 2017; 12:475-7.

7. Schleifenbaum S, Prietzel T, Aust G, Boldt A, Fritsch S, Keil I, et al. Acellularization-induced changes in tensile properties are organ specific - an in vitro mechanical and structural analysis of porcine soft tissues. PLoS One 2016;11(3): 0151223.

8. Wainwright DJ. Use of an acellular allograft dermal matrix (AlloDerm) in the management of full-thickness burns. Burns 1995;21:243-8.

9. Salzberg CA, Ashikari AY, Koch RM, Chabner-Thompson E. An 8-year experience of direct-to-implant immediate breast reconstruction using human acellular dermal matrix (AlloDerm). Plast
Reconstr Surg 2011;127:514-24.

10. Nahabedian MY. Acellular dermal matrices in primary breast reconstruction: principles, concepts, and indications. Plast Reconstr Surg 2012;130: 44S-53S.

11. Hammer N. Site-dependent acellularisation effects explain altered tissue mechanics: ultrastructural insights. Folia Morphol (Warsz) 2017;76:355-60.

12. Lugo LM, Lei $P$, Andreadis ST. Vascularization of the dermal support enhances wound re-epithelialization by in situ delivery of epidermal keratinocytes. Tissue Eng Part A 2011;17: 665-75.

13. Capito AE, Tholpady SS, Agrawal H, Drake DB, Katz AJ. Evaluation of host tissue integration, revascularization, and cellular infiltration within various dermal substrates. Ann Plast Surg 2012;68:495-500.

14. Cherubino M, Pellegatta I, Tamborini F, Cerati M, Sessa F, Valdatta L. Evaluation of lymphangiogenesis in acellular dermal matrix. Indian J Plast Surg 2014; 47:318-24.

15. Menon NG, Rodriguez ED, Byrnes CK, Girotto JA, Goldberg NH, Silverman RP. Revascularization of human acellular dermis in full-thickness abdominal wall reconstruction in the rabbit model. Ann Plast Surg 2003;50:523-7.

16. Garcia O Jr, Scott JR. Analysis of acellular dermal matrix integration and revascularization following tissue expander breast reconstruction in a clinically relevant large-animal model. Plast Reconstr Surg 2013;131:741e-51e.

17. Iyyanki TS, Dunne LW, Zhang Q, Hubenak J, Turza KC, Butler CE. Adipose-derived stem-cell-seeded noncross-linked porcine acellular dermal matrix increases cellular infiltration, vascular infiltration, and mechanical strength of ventral hernia repairs. Tissue Eng Part A 2015;21:475-85.

18. Bullocks JM. DermACELL: a novel and biocompatible acellular dermal matrix in tissue expander and implantbased breast reconstruction. Eur J Plast Surg 2014;37:529-38.

19. Dragúňová J, Kabát $\mathrm{P}$, Babál $\mathrm{P}$, Mrázová $\mathrm{H}$, Boháč M, Krajčíová L, et al. Development of a new method for the preparation of an acellular allodermis, quality control and cytotoxicity testing. Cell Tissue Bank 2017;18:153-66.

20. Mendenhall SD, Anderson LA, Ying J, Boucher KM, Liu T, Neumayer LA, et al. The BREASTrial: stage I. Outcomes from the time of tissue expander and acellular dermal matrix placement to definitive reconstruction. Plast Reconstr Surg 2015;135:29e-42e. 
21. Gubitosi A, Docimo G, Parmeggiani D, Pirozzi R, Vitiello C, Schettino P, et al. Acellular bovine pericardium dermal matrix in immediate breast reconstruction after Skin Sparing Mastectomy. Int J Surg 2014;12(Suppl 1):205-8.

22. Wong AK, Schonmeyr B, Singh $P$, Carlson DL, Li S, Mehrara BJ.
Histologic analysis of angiogenesis and lymphangiogenesis in acellular human dermis. Plast Reconstr Surg 2008;121: 1144-52.

23. Song G, Wu Y, Wang F, Shao Y, Jiang J, Fan C, et al. Development and preparation of a low-immunogenicity porcine dermal scaffold and its biocompatibility assessment. J Mater Sci Mater Med 2015;26:170.

24. Carruthers CA, Dearth CL, Reing JE, Kramer CR, Gagne DH, Crapo PM, et al. Histologic characterization of acellular dermal matrices in a porcine model of tissue expander breast reconstruction. Tissue Eng Part A 2015;21:35-44. 